\title{
Secondary skin neoplasms in patients after autologous and allogeneic hematopoietic stem cell transplantation procedures
}

\author{
Anastazja Szlauer-Stefańska ${ }^{1, A-F}$, Grażyna Kamińska-Winciorek ${ }^{1, A, B, D-F}$, Sebastian Giebel ${ }^{1, D-F}$, Maciej Bagłaj ${ }^{2, D-F}$ \\ ${ }^{1}$ Department of Bone Marrow Transplantation and Oncohematology, Maria Sklodowska-Curie Institute - Oncology Center Gliwice Branch, Poland \\ 2 Department of Pediatric Surgery and Urology, Wroclaw Medical University, Poland \\ A - research concept and design; B - collection and/or assembly of data; C - data analysis and interpretation; \\ $D$ - writing the article; $E$ - critical revision of the article; $F$ - final approval of the article
}

\section{Address for correspondence \\ Grażyna Kamińska-Winciorek}

E-mail:dermatolog.pl@gmail.com

Funding sources

None declared

Conflict of interest

None declared

Received on July 22, 2020

Reviewed on July 29, 2020

Accepted on August 25, 2020

Published online on October 16, 2020

\begin{abstract}
The increasing number of hematopoietic stem cell transplantation (HSCT) procedures and lower transplantrelated mortality has led to a growing population of survivors facing long-term increased risk of secondary malignancy, including cutaneous neoplasms. In this review, we aim to discuss the incidence, risk factors and preventive strategies for secondary skin neoplasms after autologous and allogeneic HSCT. Cutaneous neoplasms, such as basal cell carcinoma, squamous cell carcinoma and melanoma, are among the most common solid cancers arising in patients after HSCT. Besides risk factors established in the general population, primary disease, chronic graft-versus-host disease (CGvHD), prolonged immunosuppression, especially with the use of cyclosporine and azathioprine, radiation exposure, light skin color, male sex, and young age at transplantation play a role in the development of cutaneous neoplasms in HSCT recipients. Skin cancer development after HSCT may be explained by cumulative effects of chemotherapy and radiotherapy-induced DNA damage, prolonged immunosuppressive conditions and chronic mucosal inflammation, particularly after allogeneic HSCT. Delayed immune recovery and persistent immunodeficiency in patients with graftversus-host disease (GvHD) may also contribute to carcinogenesis. Regular dermatological surveillance and prompt recognition of precancerous and cancerous lesions is crucial for patient's prognosis and management.
\end{abstract}

Key words: hematopoietic stem cell transplantation, skin neoplasms, graft-versus-host disease, basal cell carcinoma, squamous cell carcinoma

Cite as

Szlauer-Stefańska A, Kamińska-Winciorek G, Giebel S, Bagłaj M. Secondary skin neoplasms in patients after autologous and allogeneic hematopoietic stem cell transplantation procedures. Adv Clin Exp Med. 2020;29(10):1221-1230. doi:10.17219/acem/126739

DOI

10.17219/acem/126739

\section{Copyright}

Copyright by Author(s)

This is an article distributed under the terms of the

Creative Commons Attribution 3.0 Unported (CC BY 3.0)

(https://creativecommons.org/licenses/by/3.0/) 


\section{Introduction}

Hematopoietic stem cell transplantation (HSCT) is potentially curative for malignant hematological neoplasms and other non-malignant conditions. The introduction of new protocols has led to the improvement of survival, ${ }^{1}$ but challenges connected with long-term health problems of patients have emerged. ${ }^{2}$ Secondary neoplasms are divided into 3 types: post-transplant lymphoproliferative disease (PTLD), hematologic malignancies and solid cancers. ${ }^{3}$ Posttransplant lymphoproliferative and hematologic diseases occur earlier after a transplant and their incidence stabilizes after 10 years, whereas solid malignancies are characterized by long latency period and no plateau even after 15 years. $^{4-6}$

In this review, we aim to discuss the incidence, risk factors and preventive strategies for secondary skin neoplasms after autologous (autoHSCT) and allogeneic hematopoietic stem cell transplantation (alloHSCT). Additionally, we present clinical and dermoscopic aspects of secondary skin malignancy in patients hospitalized in our Bone Marrow Transplantation Department (Fig. 1-5).

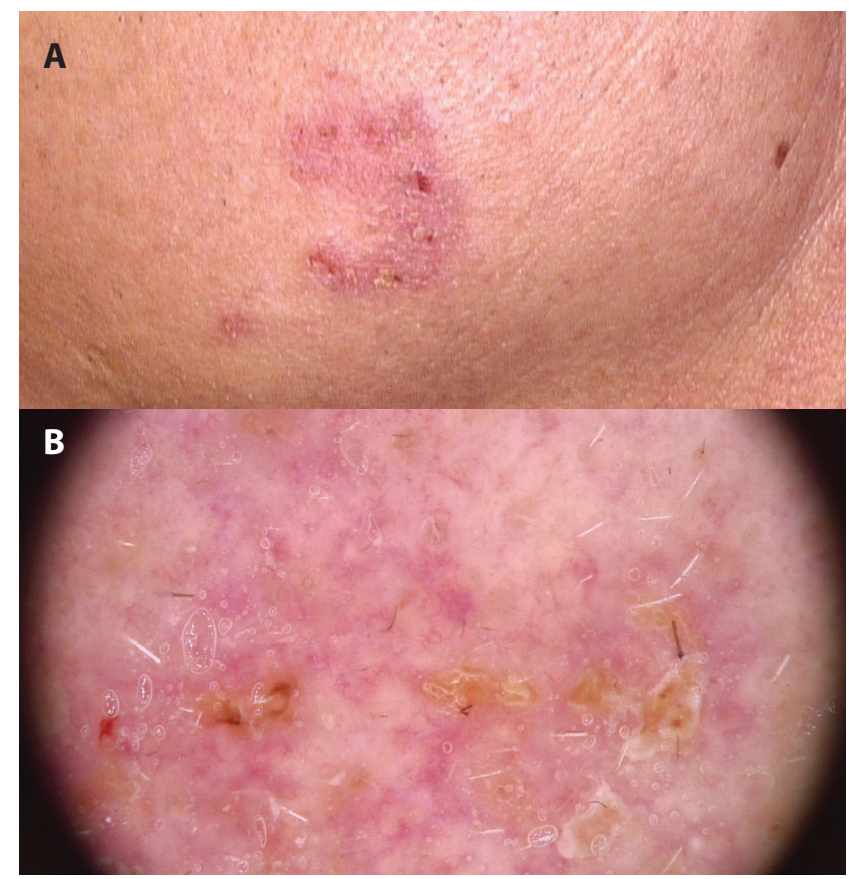

Fig. 1. Superficial variant of basal cell carcinoma (BCC) in a 52-year old male patient in +122 day after allogeneic hematopoietic stem cell transplantation (alloHSCT) for myelofibrosis. A. Clinical image of BCC presenting as a large, erythematous plaque with superficial scaling and multiple small erosions covered with crusts. B. Videodermoscopy in non-polarized light with 50-fold magnification showed the presence of multiple thin, short teleangiectasias with numerous small erosions covered with yellowish crusts

\section{Incidence of secondary skin neoplasms}

Studies usually reported incidence rate and a standardized incidence ratio (SIR) (observed cancer cases in a HSCT cohort to expected cancer cases in the general population of similar age and gender). Table 1 shows data on secondary solid neoplasms including skin neoplasms reported in analyzed studies. ${ }^{4,5,7-31}$ Secondary solid tumors have been reported to appear at twice the rate expected in the general population, ${ }^{5,19}$ although some reports cited over 11 -fold heightened risk. ${ }^{7}$ Cumulative incidence of secondary solid cancers varies from $1 \%$ to $15.9 \%$ at 10 years, ${ }^{22,25}$ reaching $17.6 \%$ at 30 years. $^{29}$

Cutaneous malignant neoplasms are among the most common neoplasms, accounting for $0-58.5 \%$ of secondary neoplasms. ${ }^{32}$ Twenty-year cumulative incidence is $6.5 \%$ for basal cell carcinoma (BCC) and 3.4\% for cutaneous squamous cell carcinoma (SCC). ${ }^{15}$ The median time from HSCT to diagnosis is 7.3-9.4 years for $\mathrm{BCC}^{13,15,17,23}$ and 2.1-7.0 years for SCC. ${ }^{11,13,15,17,19,23}$ Half of the reported melanomas occurred after $1-4$ years $^{19}$ and SIRs of melanoma are between 3.5-8.3..$^{4,14,19}$

\section{Risk factors}

The pathogenesis of secondary neoplasms after HSCT is multifactorial. Numerous risk factors have been proposed and distinct pathways may be involved in the pathogenesis of different solid tumors. ${ }^{33}$

\section{Patient-related factors}

There are conflicting results on the impact of age at transplantation. Younger patients were reported to be at risk, ${ }^{5}$ especially when irradiation-based conditioning was used. $6,15,18,19$ Old age entailed higher risk, especially in the setting of autoHSCT.4,13,25,26

Notable difference in skin cancer incidence was noted in the Asian population; some of the studies did not report on skin cancer after auto- or alloHSCT, ${ }^{12}$ despite high incidence of other solid cancers, including SCC in oral cavity. ${ }^{12}$ However, in other studies SIRs for skin cancers were reported as high as 7.2-40.23. ${ }^{23,27}$ Those discrepancies are consistent with low background incidence of skin malignancies in local cancer epidemiology and may be partly explained by gene-environment interaction. ${ }^{12}$

Genetically determined skin pigmentation plays an important role in BCC susceptibility and light complexion was reported as a risk factor in some of the analyzed studies. ${ }^{18,25,30}$ Male sex was also shown to be a risk factor in some studies. ${ }^{5,19,26}$

\section{Primary disease-related factors}

Diagnosis of Fanconi anemia, dyskeratosis congenita or Li-Fraumeni syndrome confers an increased risk of secondary cancers, also in the setting of HSCT. ${ }^{34}$ In malignant hematologic neoplasms patients, acute myeloid leukemia/myelodysplastic syndrome patients were found to have a tendency towards the development of secondary 

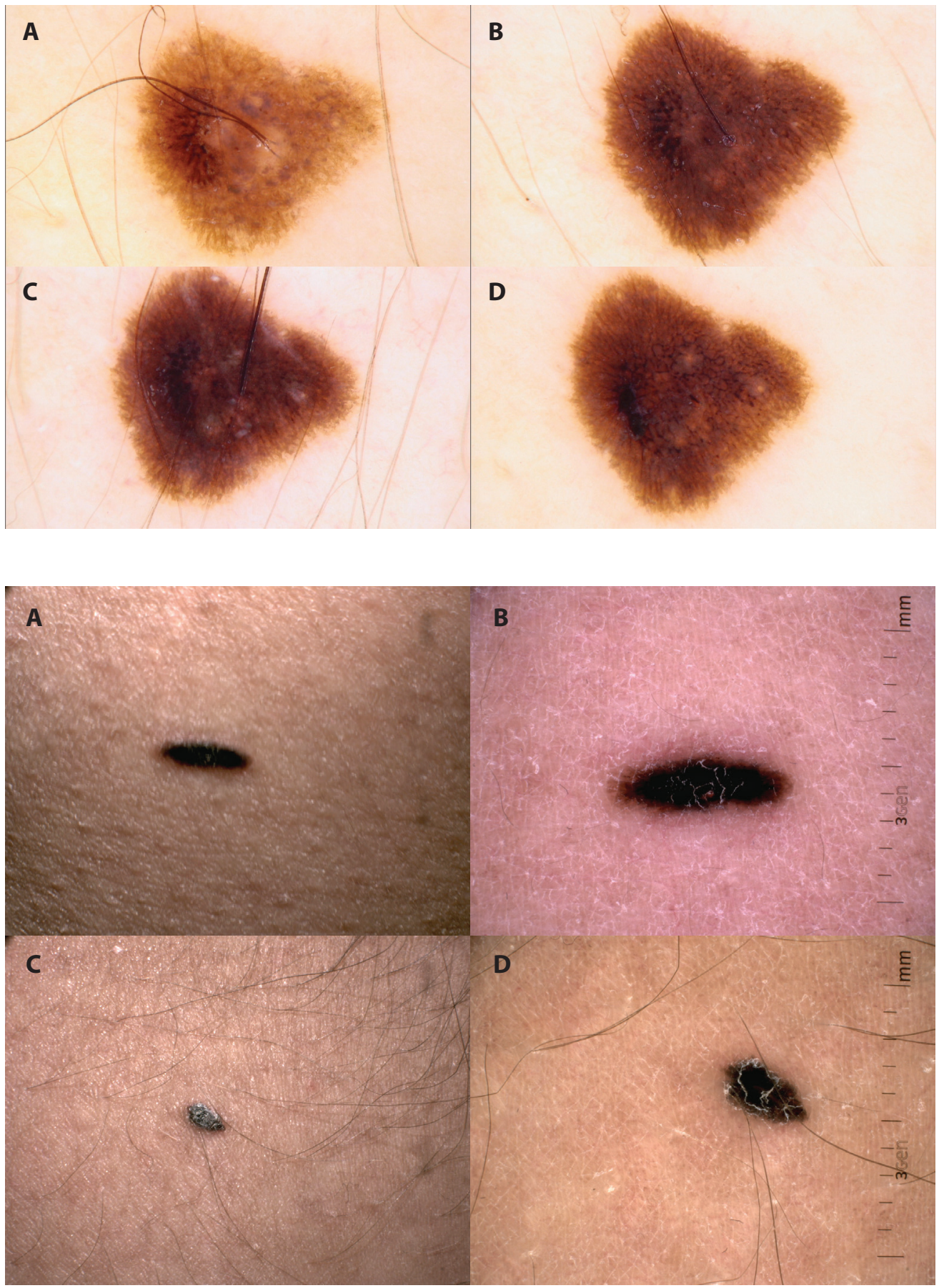

Fig. 2. Dermoscopy in non-polarized light of the monitored melanocytic nevus located on the abdominal skin in a 20-year-old female patient (III phototype of the skin according to the Fitzpatricks scale), treated with alloHSCT procedure for myelodysplastic syndrome (videodermoscopy, non-polarized light, $\times 50$ magnification)

A. Just before alloHSCT: during skin examination the melanocytic nevus $7 \mathrm{~mm}$ in diameter was found on the abdominal region. The nevus was included in the close short-time follow-up because of the presence of atypical network: sharply cut-off, with thickening of the pigmented network and presence of irregularly distributed grayish globules.

B-D. Dynamic changes observed after the alloHSCT procedure (days $+50,+72,+95)$ : pigmented network has become darker, irregularly thickened, forming peripheral short streaks and structureless irregularly distributed areas. In adhesive tape test, the black lamella was not torn off. The complete excision was postponed due to immunosuppression, agranulocytosis and thrombocytopenia. Finally, histopathologic examination revealed the diagnosis of compound nevus

Fig. 3. Macroscopic (A, C) and dermoscopic $(B, D)$ images of melanoma simulators- synchronously appearing multiple, small dark nevi in male 21-year-old patient with the IV phototype according to Fitzpatrick as a symptom of the aggravated nevogenesis after alloHSCT (day +184) for ALL. Dermoscopy in non-polarized light revealed multiple, small dark nevi with structureless dermoscopic pattern

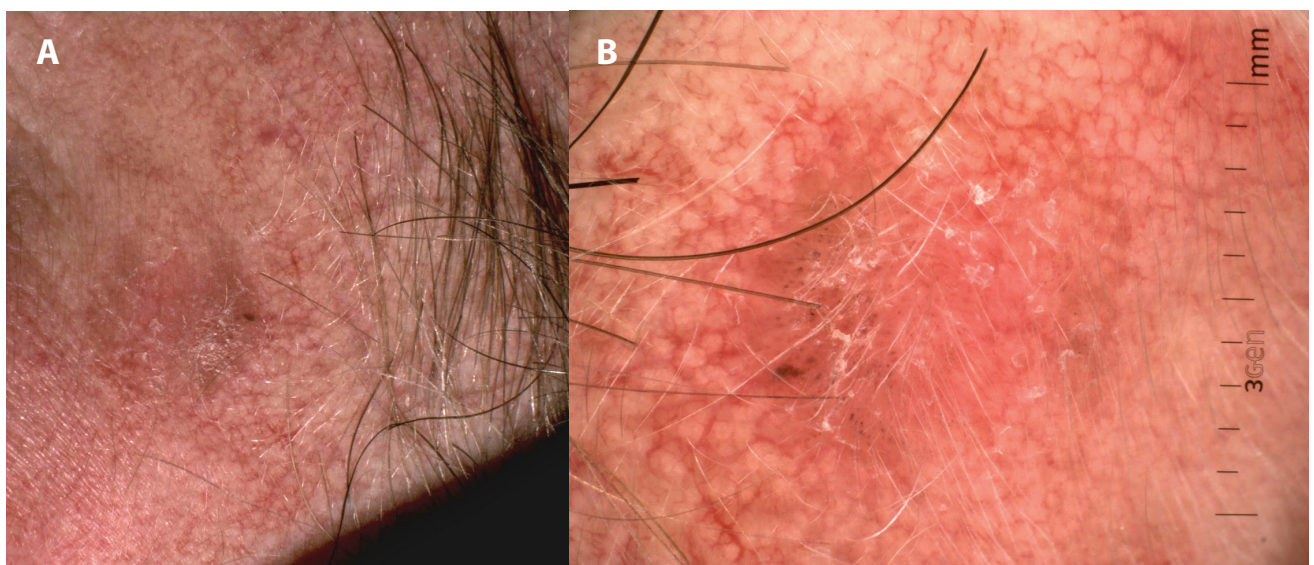

Fig. 4. Clinical and dermoscopic aspect of histopathologically confirmed squamous cell carcinoma in situ in a 54-year-old patient with cutaneous chronic GvHD after alloHSCT for myelofibrosis (day +750). A. Suspicious erythematous, velvety solitary skin lesion.

B. Dermoscopy in polarized light showed the presence of central scaling with prominent brownish dots distributed linearly at the periphery of the lesion. Background consisted of multiple linear curved vessels forming a pseudonetwork, suggesting the diagnosis of actinic keratosis 


\begin{tabular}{|c|c|c|c|c|c|c|c|c|c|c|c|c|c|}
\hline 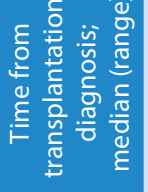 & 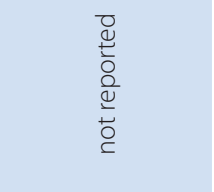 & 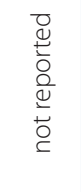 & 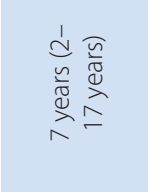 & 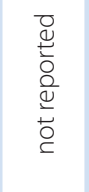 & 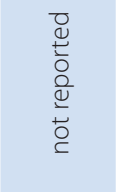 & 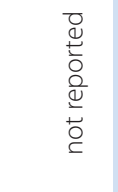 & 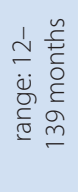 & 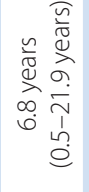 & 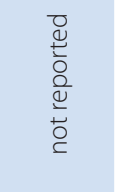 & 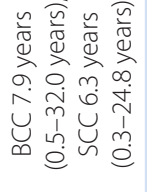 & 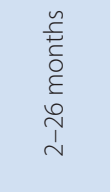 & 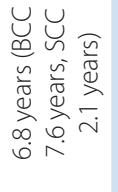 & 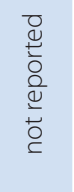 \\
\hline 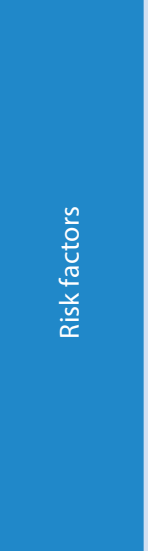 & 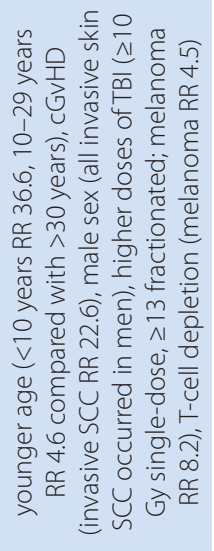 & 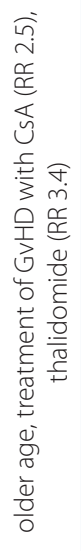 & 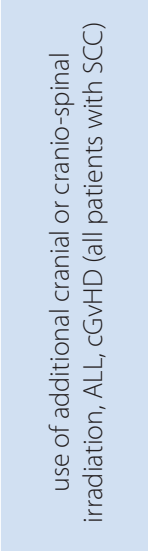 & 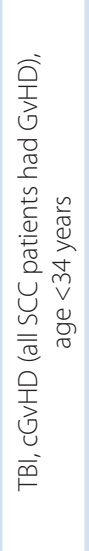 & 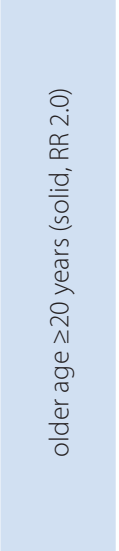 & 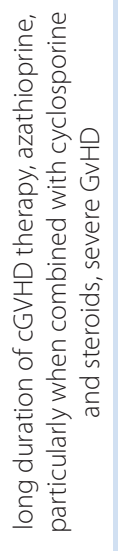 & 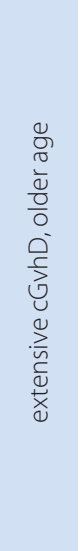 & $\begin{array}{l}\stackrel{\Xi}{\Phi} \\
\overline{\bar{z}} \\
\frac{\bar{y}}{0}\end{array}$ & $\begin{array}{l}\text { ठू } \\
\bar{\Xi} \\
\frac{\bar{g}}{0}\end{array}$ & 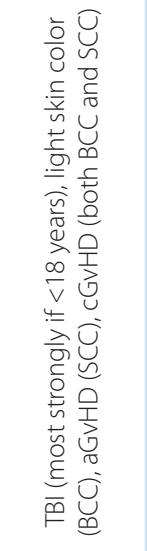 & 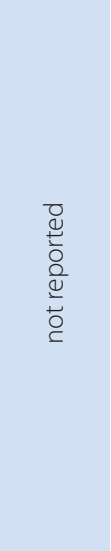 & 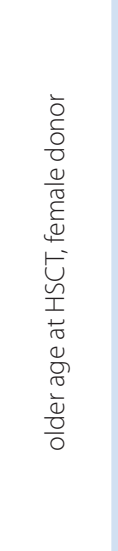 & 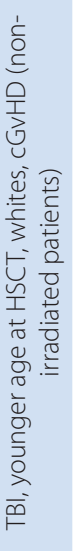 \\
\hline 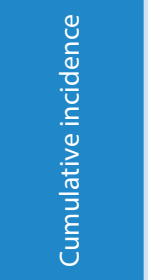 & 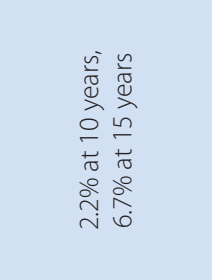 & 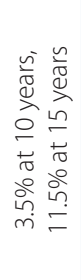 & 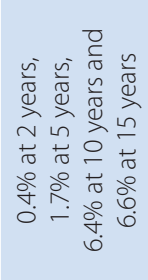 & 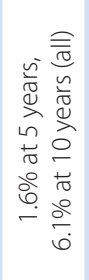 & 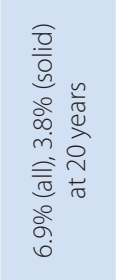 & 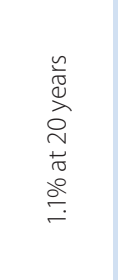 & 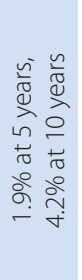 & 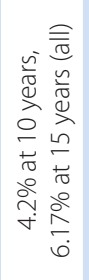 & 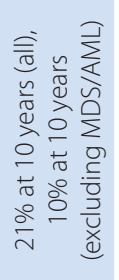 & 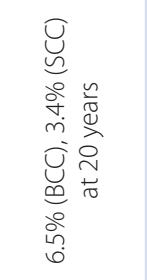 & $\begin{array}{l}\overline{0} \\
\frac{0}{0} \\
\frac{0}{0} \\
\frac{0}{2} \\
\stackrel{0}{c}\end{array}$ & 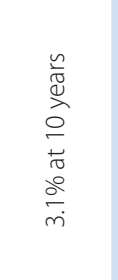 & 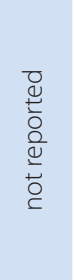 \\
\hline 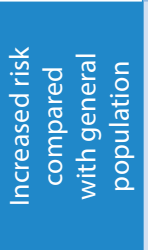 & 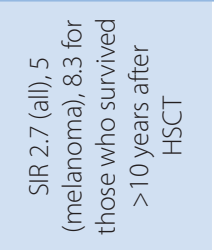 & $\begin{array}{l}\stackrel{\infty}{m} \\
\stackrel{\sim}{ज}\end{array}$ & 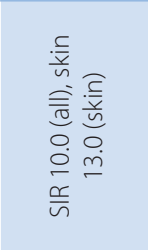 & 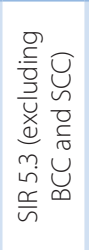 & 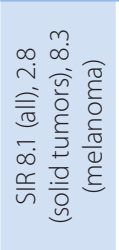 & & 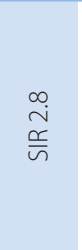 & $\begin{array}{l}\text { 产 } \\
m \\
\stackrel{m}{i n} \\
\frac{n}{n}\end{array}$ & 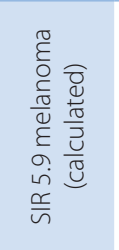 & 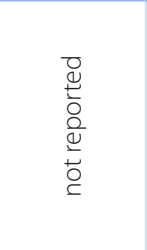 & 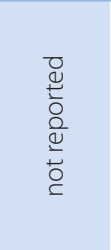 & $\begin{array}{l}\stackrel{\omega}{\infty} \\
\dot{\infty} \\
\frac{\infty}{n}\end{array}$ & 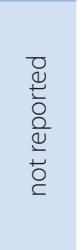 \\
\hline 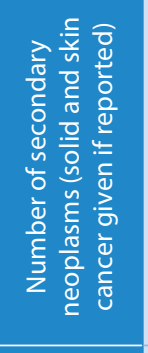 & 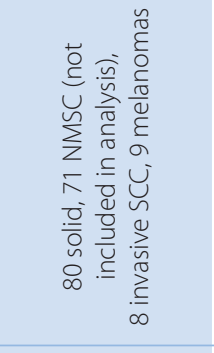 & 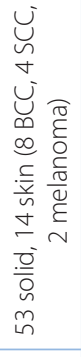 & 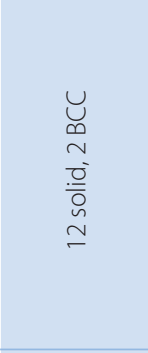 & 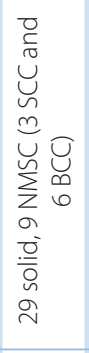 & 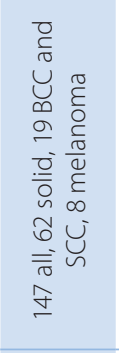 & 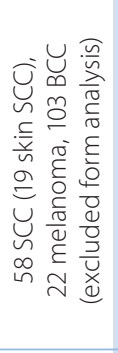 & 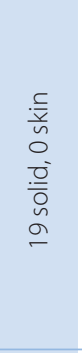 & 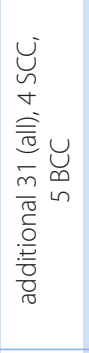 & 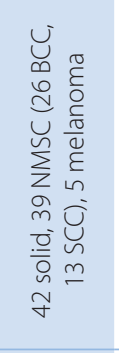 & 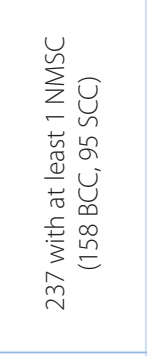 & 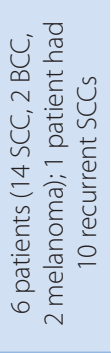 & 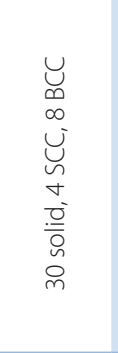 & 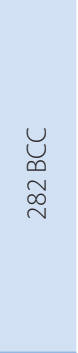 \\
\hline 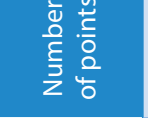 & $\begin{array}{l}\text { సે } \\
\stackrel{\sigma}{\sigma}\end{array}$ & ஜ̊లి & $\stackrel{\aleph}{\aleph}$ & $\underset{\sim}{\stackrel{\sim}{\sim}}$ & $\underset{\text { N }}{\stackrel{N}{n}}$ & 高 & هे & 会 & $\stackrel{n}{8}$ & $\stackrel{\circ}{\stackrel{\circ}{o g}}$ & $\stackrel{g}{\sigma}$ & ฉू & 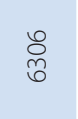 \\
\hline 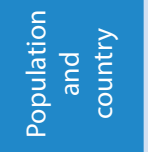 & 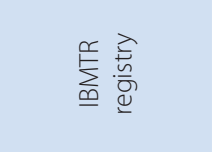 & 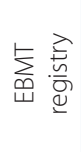 & 兰 & 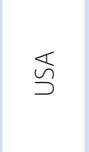 & 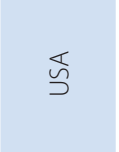 & 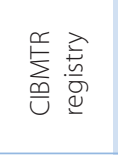 & $\begin{array}{l}\frac{1}{\pi} \\
\substack{0\\
}\end{array}$ & 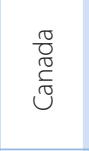 & 崩 & 崩 & 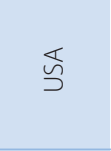 & 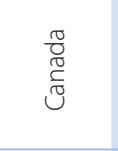 & 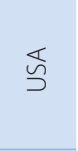 \\
\hline 总点 & $\stackrel{\circ}{\bar{\sigma}}$ & 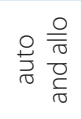 & $\stackrel{\circ}{\bar{\sigma}}$ & 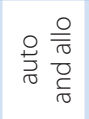 & 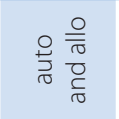 & $\stackrel{\circ}{\frac{0}{\sigma}}$ & 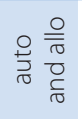 & $\stackrel{\circ}{\frac{0}{\sigma}}$ & 疍 & $\stackrel{\circ}{\bar{\sigma}}$ & $\stackrel{\circ}{\bar{\sigma}}$ & $\stackrel{\circ}{\frac{\circ}{\sigma}}$ & 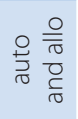 \\
\hline 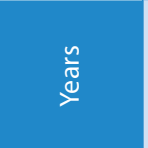 & 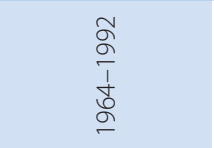 & 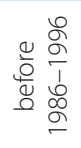 & $\begin{array}{l}\hat{\sigma} \\
\sigma \\
\overline{1} \\
\hat{\sigma} \\
\sigma\end{array}$ & 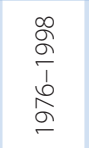 & 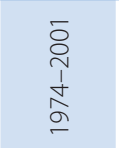 & 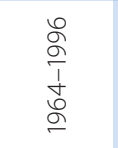 & $\begin{array}{l}\stackrel{8}{0} \\
\stackrel{1}{1} \\
\frac{1}{\infty} \\
\stackrel{0}{-}\end{array}$ & 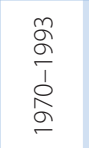 & $\begin{array}{l}\hat{\sigma} \\
\stackrel{1}{1} \\
\alpha \\
o \\
\sigma\end{array}$ & 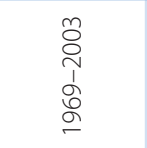 & 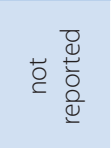 & 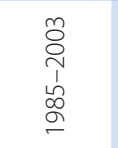 & 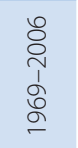 \\
\hline 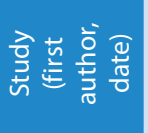 & 芞瓷 & $\begin{array}{l}\frac{\alpha}{\alpha} \\
\frac{1}{2} \\
\frac{0}{0}\end{array}$ & 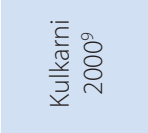 & 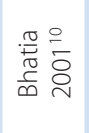 & 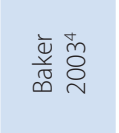 & 岂言 & 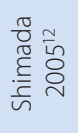 & 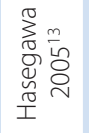 & 戈 & 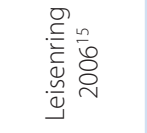 & 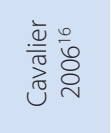 & 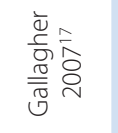 & 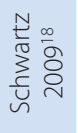 \\
\hline
\end{tabular}




\begin{tabular}{|c|c|c|c|c|c|c|c|c|c|c|c|c|c|}
\hline 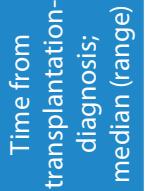 & 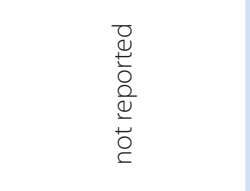 & 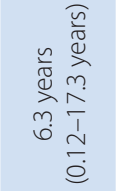 & 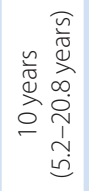 & $\begin{array}{l}\frac{n}{\widetilde{J}} \\
\stackrel{2}{\sigma}\end{array}$ & 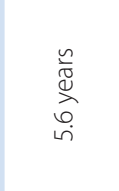 & 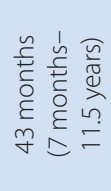 & 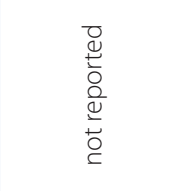 & 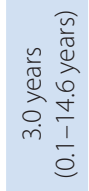 & 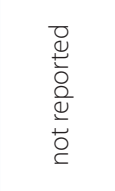 & 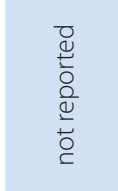 & 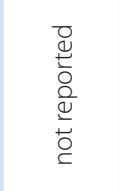 & 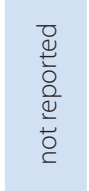 & 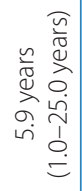 \\
\hline 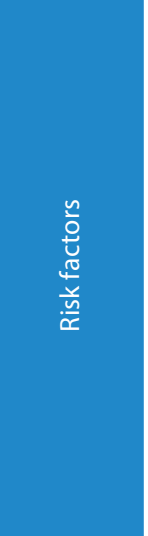 & 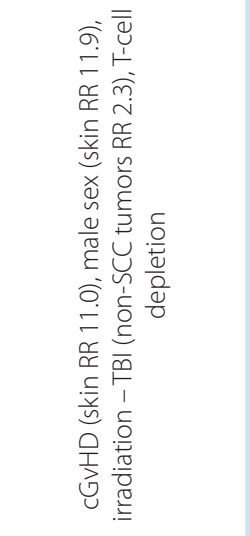 & 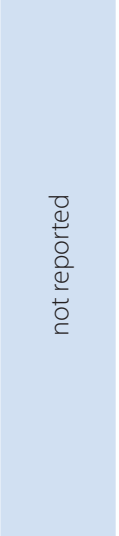 & 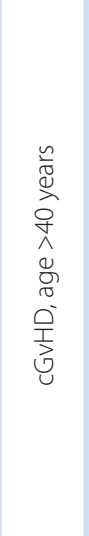 & 옳 & 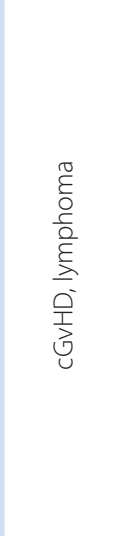 & 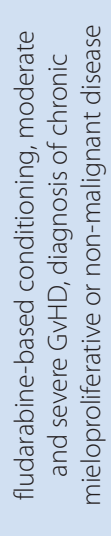 & 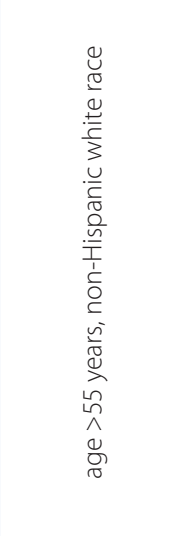 & 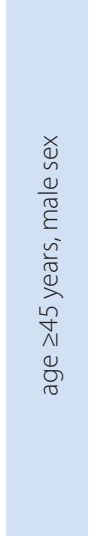 & 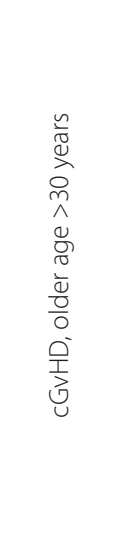 & 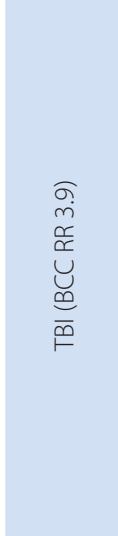 & 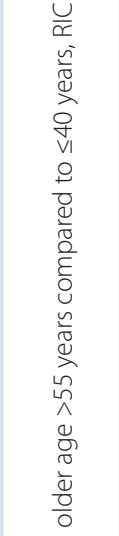 & 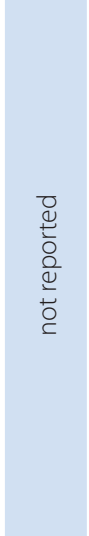 & 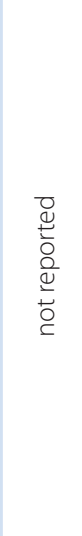 \\
\hline 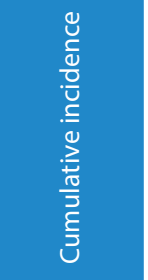 & 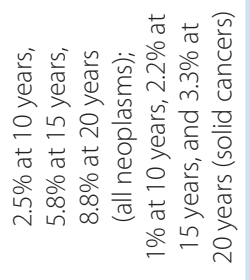 & 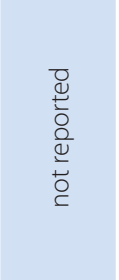 & 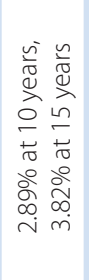 & 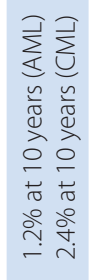 & 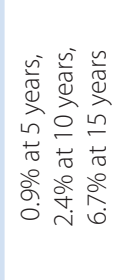 & 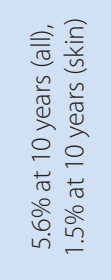 & 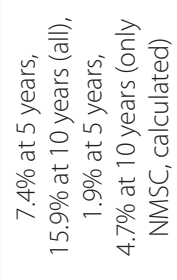 & 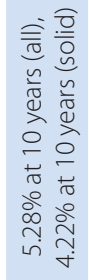 & 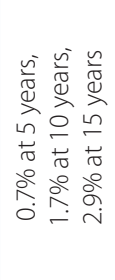 & 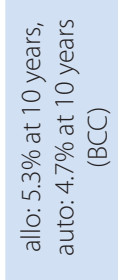 & 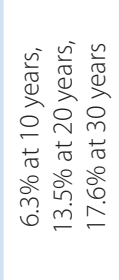 & 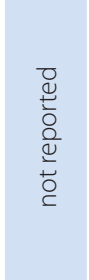 & 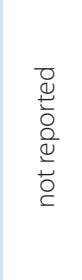 \\
\hline 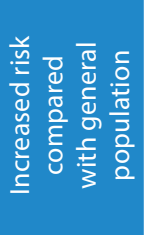 & 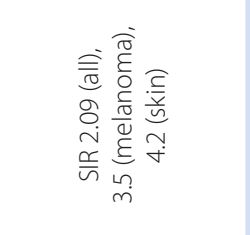 & 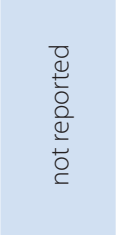 & 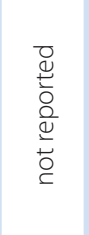 & 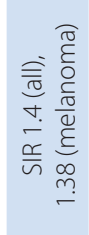 & 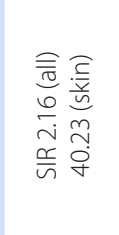 & 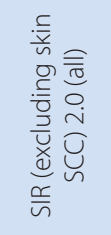 & 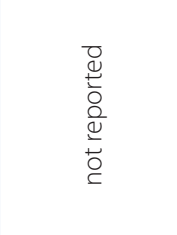 & 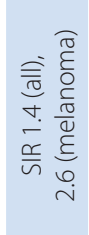 & 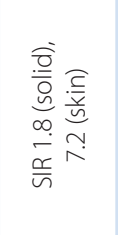 & 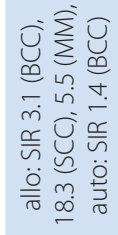 & 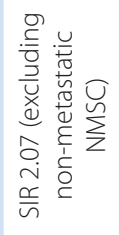 & 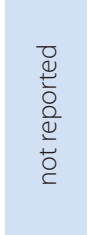 & 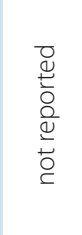 \\
\hline 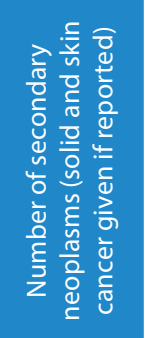 & 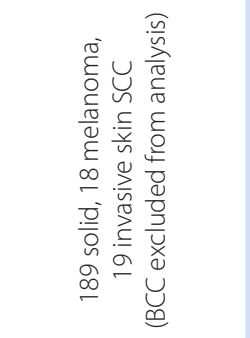 & 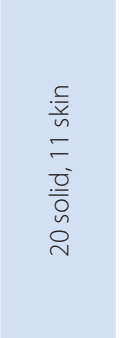 & 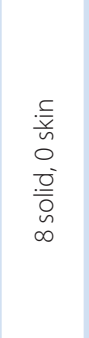 & 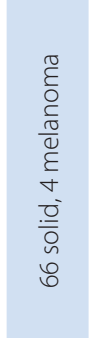 & 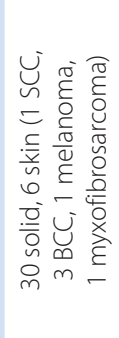 & 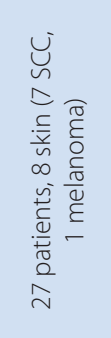 & 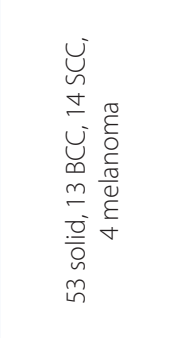 & 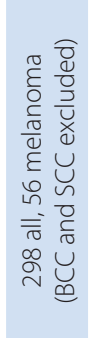 & 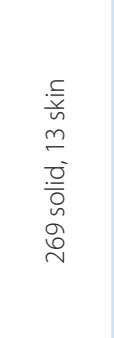 & 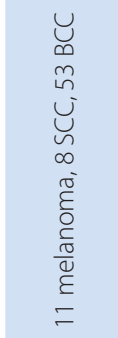 & 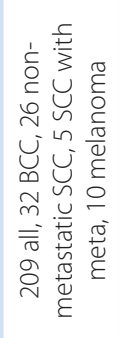 & 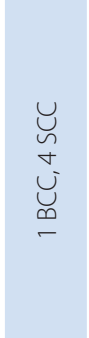 & 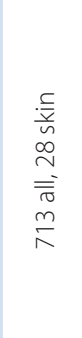 \\
\hline 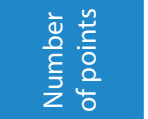 & 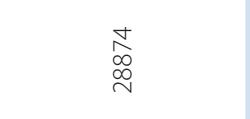 & શે & $\stackrel{尺}{\stackrel{2}{2}}$ & $\stackrel{\infty}{\bar{\gamma}}$ & ర్రి & $\bar{\alpha}$ & $\bar{\infty}$ & $\stackrel{\stackrel{n}{\circ}}{\stackrel{2}{2}}$ & 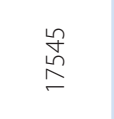 & ָ̊ & $\stackrel{\stackrel{n}{\sigma}}{\sim}$ & $\mathscr{L}_{\infty}^{n}$ & $\frac{\infty}{\infty}$ \\
\hline 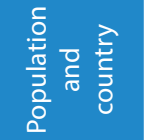 & 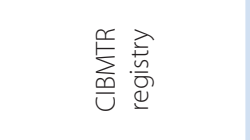 & $\begin{array}{l}\frac{\pi}{0} \\
\mathbb{\pi} \\
\mathbb{J}\end{array}$ & 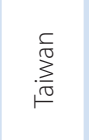 & 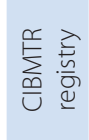 & $\begin{array}{l}\frac{1}{\sqrt[0]{0}} \\
\frac{0}{\pi}\end{array}$ & $\begin{array}{l}\overline{\bar{q}} \\
\underline{\underline{\tilde{N}}}\end{array}$ & 㤐 & 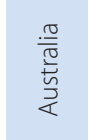 & 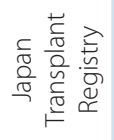 & 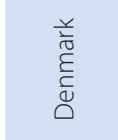 & $\begin{array}{l}\frac{\pi}{0} \\
\substack{\pi \\
ٍ}\end{array}$ & 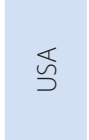 & $\begin{array}{l}\frac{1}{0} \\
\frac{0}{0} \\
\frac{0}{n}\end{array}$ \\
\hline 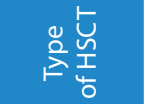 & $\stackrel{\circ}{\bar{\sigma}}$ & $\stackrel{\circ}{\bar{\sigma}}$ & $\stackrel{\circ}{\bar{\sigma}}$ & $\stackrel{\circ}{\frac{0}{\sigma}}$ & $\stackrel{\circ}{\bar{\sigma}}$ & $\stackrel{\circ}{\bar{\sigma}}$ & 疍 & 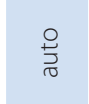 & $\stackrel{\circ}{\bar{\sigma}}$ & 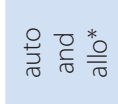 & $\stackrel{\circ}{\bar{\sigma}}$ & $\stackrel{\circ}{\frac{\circ}{\sigma}}$ & 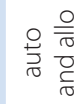 \\
\hline 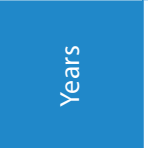 & \begin{tabular}{l} 
\& \\
$\frac{1}{1}$ \\
$\frac{1}{0}$ \\
\hdashline
\end{tabular} & $\begin{array}{l}\widetilde{0} \\
\stackrel{1}{1} \\
\frac{1}{\infty} \\
\stackrel{0}{\circ}\end{array}$ & 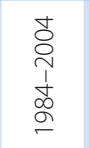 & 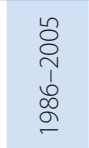 & 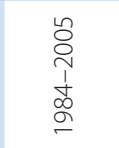 & 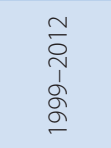 & 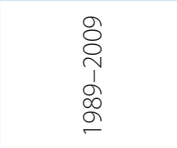 & 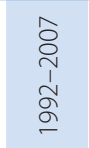 & 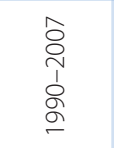 & 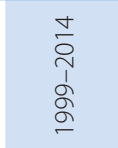 & 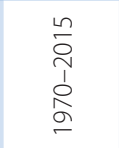 & \begin{tabular}{l}
$m$ \\
\multirow{2}{*}{} \\
$\frac{1}{\alpha}$ \\
$o$
\end{tabular} & $\begin{array}{l}m \\
\stackrel{n}{a} \\
\vdots \\
o \\
\circ\end{array}$ \\
\hline 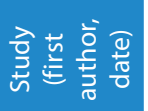 & 离 & 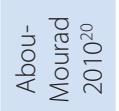 & 这 & 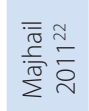 & 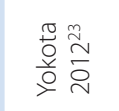 & 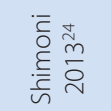 & 总 & 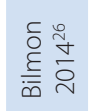 & 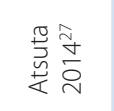 & 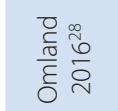 & 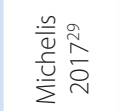 & 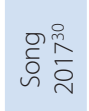 & 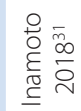 \\
\hline
\end{tabular}




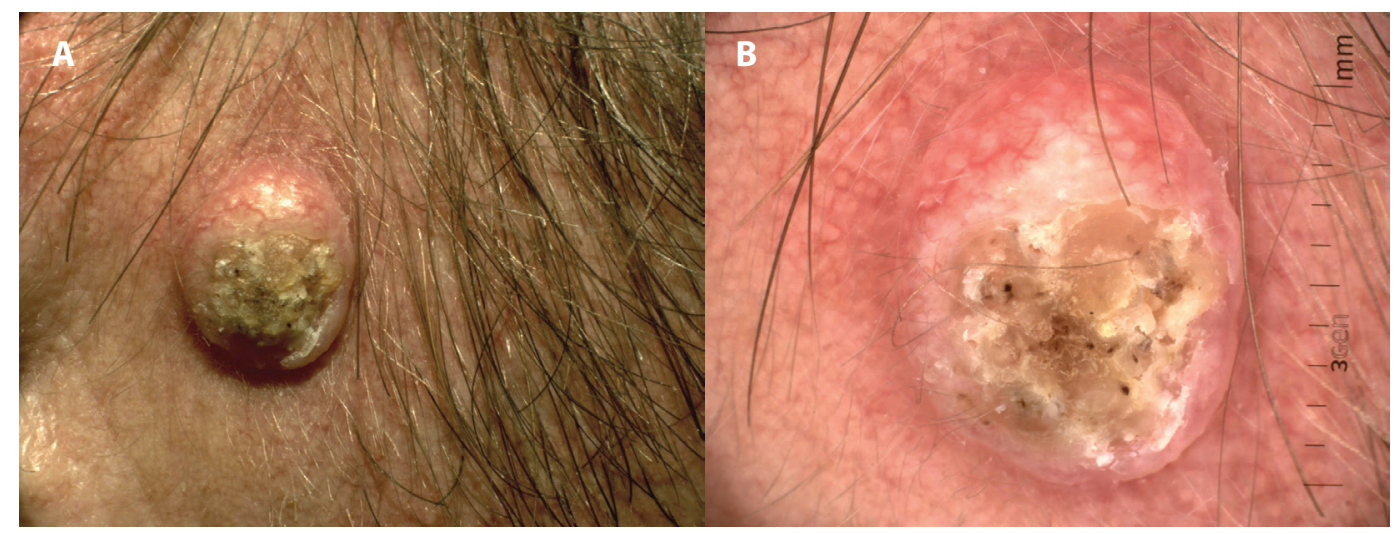

Fig. 5. Raised skin lesion in a 54-year-old male patient suffering from cutaneous chronic GvHD after alloHSCT for myelofibrosis (day +750 ). Histopathologic examination after total surgical excision of the presented lesion confirmed the diagnosis of keratoacanthoma. A. A rapidly growing skin lesion appeared within a one-month period as a dome-shaped $1 \mathrm{~cm}$ nodule with the central keratin masses. B. Dermoscopy in polarized light revealed the presence of central keratin masses with several blood spots and several branched serpentine vessels located at the periphery

neoplasms. ${ }^{16}$ Multiple myeloma was also linked with secondary neoplasms. ${ }^{25,26}$ Rizzo et al. reported lower risk for patient with chronic myeloid leukemia compared to acute leukemia, ${ }^{19}$ but this claim was not confirmed in other studies. $^{20}$

\section{Transplantation-related factors}

Initially, in the HSCT procedure myeloablative conditioning using total body irradiation (TBI) or high-dose alkylating agents was performed. Presently, non-myeloablative conditioning that utilizes transient intensive immunosuppression enabling engraftment of allogeneic material without myeloablation is frequently used. Reduced intensity and toxicity conditioning (RIC/RTC) were also developed. The incidence of secondary neoplasms is not lower in the setting of non-myeloablative or $\mathrm{RIC}^{16,24}$; in some reports, the incidence was even higher. ${ }^{29}$ Presently, the widespread use of haploidentical transplantation with post-transplant cyclophosphamide is noted, but there is scarce data on secondary neoplasms in this population.

All alkylating agents are considered carcinogenic, and the effect varies among the drugs in this group. There is concern regarding the high neogenic potential of melphalan; however, its use in conditioning was not associated with a higher risk of second malignancies than cyclophosphamide and TBI. ${ }^{9}$ Also, neither cyclophosphamide, busulfan conditioning, cyclophosphamide-based mobilizing therapy, nor epipodophyllotoxins were found to increase the risk of secondary neoplasms in autoHSCT recipients treated for multiple myeloma. ${ }^{25}$ Fludarabine is associated with the risk of secondary malignancies, ${ }^{24}$ as its active anti-metabolite incorporates into DNA, resulting in the inactivation and inhibition of DNA repair. It is speculated that the combination of fludarabine with alkylating agents as given in RIC/RTC may have a synergistic carcinogenic effect. ${ }^{24}$

\section{Radiation}

The impact of radiation seems to depend on the age of exposure; younger people were reported to have almost a ten-fold higher risk of secondary neoplasms, while for older patients it was only slightly elevated. ${ }^{19}$ In a study focusing on BCC, TBI was reported as a risk factor, especially in patients $<18$ years, with relative risk exceeding 20 for those $<10$ years and with light complexion; however, the impact declined with age and there was no increased $\mathrm{BCC}$ risk after TBI conditioning in patients over the age of $40 . .^{18}$

The risk of radiation-related malignancies and other long-term adverse effects, particularly in children, has led from fractionation and reduction of doses to the development of non-radiation-based regimens. A study involving a population of patients without TBI exposition, utilizing busulfan/cyclophosphamide, reported lower rates of secondary cancers, although still 1.4-fold higher than general population..$^{22}$ However, the role of TBI remains unresolved, with some publications providing strong evidence of increased risk from TBI exposure, ${ }^{15}$ and others failing to identify this association., ${ }^{9,20,29}$

\section{Immunosuppression and graft-versus-host disease}

After auto- and alloHSCT, a period of lymphopenia and cell-mediated immune deficiency occurs and can persist for months. Adequate numbers of CD4+ T-lymphocytes have been postulated to inhibit malignant transformation of precursor skin lesions, and their lack is reflected in the most common cancer related with AIDS - Kaposi sarcoma. ${ }^{35}$ There are also reports of telomere shortening after alloHSCT, which can cause significant genomic instability, leading to malignant transformation. ${ }^{36}$ What is more, low-grade inflammation may not be clinically perceived; in a study by Vasallo et al., "normal-looking" 
skin in $76 \%$ of patients around day 100 after HSCT showed inflammatory abnormalities in histopathological assessment. ${ }^{37}$ In the case of graft-versus-host disease (GvHD) with persistent inflammation, ulceration and scarring may itself be a risk factor for carcinogenesis. Repeated cell division correlates with chromosomal abnormalities in keratinocytes, including aneuploidy and haploinsufficiency for p53 due to loss of chromosome $17 .^{36}$

Immunosuppressed patients are at risk of developing cutaneous neoplasm ${ }^{35}$ and immunosuppression is a mainstay of GvHD treatment. Recent meta-analysis encompassing 50951 HSCT recipients showed that chronic GvHD (cGvHD) was associated with increased incidence of BCC (RR 1.95) and SCC (RR 5.31) but had no effect on melanoma and acute GvHD (aGvHD) alone was not associated with increased risk of skin cancer. ${ }^{38}$ A large case-control study of 24011 HSCT recipients ${ }^{11}$ reported that the risk of SCC was almost three-fold higher in those with cGvHD and even higher with previous aGvHD. Therapy of GvHD with azathioprine, cyclosporine, steroids, psoralen, ultraviolet A (PUVA), and others increased the risk for SCC 18 to 50 -fold. Several other studies consistently reported GvHD as a risk factor. ${ }^{16,18,27}$

Azathioprine in GvHD treatment was identified as the major risk factor for the development of secondary cancers after alloHSCT. ${ }^{11}$ Possible mechanisms include incorporation of activated azathioprine forms into DNA, making it susceptible to mutagenic oxidation, especially after radiation therapy. ${ }^{39}$ Cyclosporine, a calcineurin inhibitor, commonly used in immunosuppression after transplant, is a carcinogen causing induction of phenotypic changes and enhancement of invasiveness of cells through transforming growth factor $\beta$ (TGF- $\beta$ ) mechanisms. ${ }^{40}$ Topical pimecrolimus use was questioned due to its similarity to oral calcineurin inhibitors. In the pediatric population, it was linked to the development of precancerous and cancerous lesions. ${ }^{30}$

Voriconazole, a recommended antifungal agent both in prophylaxis and treatment, was found to increase the risk of skin cancer and precancerous lesions in HSCT recipients. ${ }^{30,41}$ A multistep mechanism of carcinogenesis involving acute phototoxicity, then actinic keratosis followed by SCC was suggested. ${ }^{42}$

\section{Oncogenic viruses}

Oncogenic viruses in the context of prolonged immunosuppression also participate in pathogenesis of tumors after HSCT. Human papillomavirus (HPV) infection was suggested to be involved in the development of non-melanoma skin cancer. ${ }^{35}$ Cancers associated with oncogenic viruses express oncoproteins inactivation $\mathrm{p} 53$, which is associated with the development of BCC and SCC. HPV DNA, especially types 5 and 8, was detected more frequently in SCC of transplant recipients than in non-immunosuppressed patients. ${ }^{43}$ However, in 1 study, none of the oral SCCs showed evidence of HPV infection. ${ }^{21}$

\section{Hematopoietic stem cell transplantation and nevi}

In a recent study comprising pediatric patients, ${ }^{30}$ the patients after HSCT had significantly more nevi and 16.5\% of HSCT recipients developed cancerous or precancerous lesions. ${ }^{30}$ The majority of nevogenesis occurs in childhood; in a study of adult and pediatric HSCT recipients, an increase in the nevus count was found only among those aged $<20$ years at HSCT. ${ }^{44}$ In addition, children may be at a higher risk of thymic dysfunction after HSCT, with impaired immunosurveillance, possibly contributing to the development of secondary malignancies and nevogenesis. ${ }^{30}$ In another study, the number of nevi was not significantly increased after HSCT, ${ }^{44}$ although a group of patients who were conditioned with a combination of 2 alkylating drugs at high doses and younger patients tended to have a higher count of nevi. Conversely, there was a trend in favor of a lower count of nevi in patients presenting with cutaneous cGvHD. Alloimmunity, chronic skin inflammation with overproduction of pro-inflammatory cytokines or pigmentation, areas of depigmentation, leukoderma, and fibrosis in cGvHD may be responsible for the perceivable decreased number of nevi, although this observation requires further investigation. ${ }^{44,45}$

\section{Differences between primary and secondary skin cancers}

Risk factors for the development of skin cancer in the general population, including fair skin type, advanced age, exposure to UV radiation, and genetic predisposition, seem to play a role in the initiation and progression of carcinogenesis in skin of HSCT recipients. ${ }^{15,18,25,30}$ However, SCC after transplantation may not share all conventional risk factors; in an evaluation of SCC of the buccal cavity, no excess risk was linked to alcohol or tobacco use. ${ }^{11}$

Secondary malignancies after alloHSCT tend to behave more aggressively in these patients than primary ones in immunocompetent individuals, and they have a higher risk of metastasis ${ }^{46}$ and are often multiple. ${ }^{47,48}$ Adjusted overall survival probabilities were lower in patients with subsequent cancer compared with those with primary cancer in the general population for colon, central nervous system and bone/ soft tissue cancers after allogeneic HSCT. ${ }^{31}$ Michelis et al. ${ }^{29}$ found that 40 of 209 patients (19\%) with secondary malignancy developed another one, including 13 patients with local skin cancers recurrences and 12 patients presenting with SCC or BCC before other solid malignancy. In the study, 22\% of long-term survivors' deaths were attributable to secondary neoplasms. Of note, 4 out of 5 metastatic cutaneous SCC carcinomas in this study were reported to be a cause of death. ${ }^{29}$

In a study by Inamoto et al., ${ }^{31}$ secondary cancers occurred in alloHSCT recipients at younger age than primary cancers in the general population (median 55 compared to 67 years). 


\section{Differences between solid organ transplantation and HSCT}

The risk of secondary skin malignancies is high in solid-organ transplant recipients and has been extensively studied. ${ }^{49}$ The incidence of cutaneous SCC in solid-organ transplant recipients is 65 - to 250 -fold greater than in the general population, and this cancer has greater morbidity and mortality in solid-organ recipients than in the general population. ${ }^{50}$ The risk factors include cumulative ultraviolet radiation exposure, long-term use of immunosuppressive agents and infections by human papillomaviruses. Several guidelines and risk prediction tools have been established for this population of patients. ${ }^{49}$ Recent research observed a reduction in cumulative incidence of secondary cancers when sirolimus was used instead of cyclosporine. ${ }^{51}$ Emerging possible chemoprophylaxis include retinoids, antioxidants, difluormethylornithine, and cyclooxygenase-2 (COX-2) inhibitors. ${ }^{49,51}$

For solid-organ transplant recipients, the duration of immunosuppressive therapy is usually lifelong, whereas in HSCT recipients, it may be discontinued after transplantation if they do not develop GvHD. Thus, prolonged immunosuppression and GvHD are usually linked. On the other hand, solid organ recipients rarely develop GvHD, which itself causes processes of tissue destruction and possible tumor development. Omland et al. compared HSCT recipients with renal transplant patients; alloHSCT recipients had a three-fold higher risk of melanoma, similar risk of $\mathrm{BCC}$ and lower risk of SCC. ${ }^{28}$

\section{Limitations of the studies}

There are several limitations in the published studies. Reports with long follow-up reflect the then used transplantation strategies, which have since greatly changed. In older reports, bone marrow as a source of stem cells and HLA identical matched sibling donor with myeloablative conditioning were predominantly used. Presently, peripheral blood cells are dominant as a graft source, and alternative conditioning regimens - RIC and haploidentical transplantations - are commonly utilized. ${ }^{52}$ Furthermore, immunosuppression strategies and treatment of GvHD have changed. Some studies reported combined results of allo- and autoHSCT, and others included a considerable number of pediatric patients among the adults, resulting in heterogeneity of population. Most established diagnoses of second neoplasms from hospital records or from patient's self-reports potentially underestimate risk, particularly in patients without other post-transplant complications. What is more, there is a lack of information on cancer stage at diagnosis, localization of lesions and treatment details.

National cancer registries rarely include information on non-melanoma skin cancer. Only some of the studies included an assessment of the risk in comparison to the general population. The majority of studies did not include non-melanoma skin cancers in the analysis, citing low mortality and unknown incidence of BCC and SCC, so it was not possible to assess excess risk, SIR or specific factors for development of secondary skin cancers. Retrospective studies lack assessment of Fitzpatrick's skin phototype and detailed patient history. No prospective study with pre-transplant skin assessment focusing on risk of skin neoplasms have been published. Other issues are long latency period necessary for the occurrence of these complications and a relatively low numbers of events. Follow-up of some of the studies may be too short to predict the actual incidence of skin cancer.

Additional studies with systematic data collection and comprehensive reporting with extended follow-up are needed to characterize the incidence and actual risk for developing skin cancer.

\section{Screening and preventive measure recommendations}

Patients after HSCT should follow the general population recommendations: avoidance of carcinogenic agents such as tobacco and alcohol, and use of sun-protection measures. Specific guidelines for prophylaxis are consensus-based and include whole skin and mucous membranes assessment by dermatologist every 12 months. ${ }^{3,53,54}$ In patients with a history of cutaneous malignancies or GvHD, screening interval should be shortened to at least 6 months. ${ }^{55}$ Patients should be educated about prevention and recognition of skin cancers. ${ }^{55}$ The role of the dermatologist in the care of HSCT recipients is important and includes also diagnosis and treatment of cutaneous GvHD; thus, the development of dedicated dermatology service for allogeneic HSCT was proposed. ${ }^{56}$ Efforts to prevent GvHD and to improve immune reconstitution after transplantation may be an effective strategy of preventing secondary tumors. During the assessment of skin lesions in HSCT recipients, it is important to consider a possible differential diagnosis that includes a plethora of GvHD manifestations, cutaneous manifestation of primary neoplasms, infectious lesions, and others.

Heightened awareness and more vigilant skin surveillance are warranted for patients with GvHD who received TBI-based conditioning, and those with hereditary disorders associated with cancer risk, such as Fanconi anemia. Discontinuation of voriconazole may be considered in patients experiencing chronic phototoxicity. Suspicious lesions should be addressed promptly, with management complying with standard practice, but the treatment plans should include previous history. ${ }^{57}$ There are no studies on specific skin cancer preventative measure in the population of patients after HSCT. It would be valuable to find whether preference of mTOR inhibitors, such 
as sirolimus, is protective, as was shown in solid-organ transplant recipients. ${ }^{51}$

Studies on HSCT recipients have reported generally high adherence rate to cancer screening; however, it was reported that autoHSCT survivors were less likely than alloHSCT to have a skin examination in the previous year. ${ }^{58}$ Physicians should have lower thresholds to investigate new concerning signs or symptoms of malignancy in patients after HSCT than for the general population. Many transplantation centers expect to receive notification if their survivors develop second cancers. ${ }^{57}$ Regular dermatological surveillance and prompt recognition of precancerous and cancerous lesions is crucial for a patient's prognosis and management.

\section{ORCID iDs}

Anastazja Szlauer-Stefańska [1] https://orcid.org/0000-0002-7256-1136 Grażyna Kamińska-Winciorek (1) https://orcid.org/0000-0002-9810-4945 Sebastian Giebel (1) https://orcid.org/0000-0002-4827-4401

Maciej Bagłaj (1) https://orcid.org/0000-0002-6291-1577

\section{References}

1. Gooley TA, Chien JW, Pergam SA, et al. Reduced mortality after allogeneic hematopoietic cell transplantation. NEng/JMed.2010;363(22): 2091-2101. doi:10.1056/NEJMoa1004383

2. Mohty $B$, Mohty M. Long-term complications and side effects after allogeneic hematopoietic stem cell transplantation: An update. Blood Cancer J. 2011;1(4):e16-e25. doi:10.1038/bcj.2011.14

3. Morton LM, Saber W, Baker KS, et al. National Institutes of Health Hematopoietic Cell Transplantation Late Effects Initiative: The Subsequent Neoplasms Working Group report. Biol Blood Marrow Transplant. 2017;23(3):367-378. doi:10.1016/j.bbmt.2016.09.005

4. Baker KS, DeFor TE, Burns LJ, Ramsay NKC, Neglia JP, Robison LL. New malignancies after blood or marrow stem cell transplantation in children and adults: Incidence and risk factors. J Clin Oncol. 2003;21(7): 1352-1358. doi:10.1200/JCO.2003.05.108

5. Curtis RE, Rowlings PA, Deeg HJ, et al. Solid cancers after bone marrow transplantation. NEnglJ Med. 1997;336(13):897-904. doi:10.1056/ NEJM199703273361301

6. Socié G, Baker KS, Bhatia S. Subsequent malignant neoplasms after hematopoietic cell transplantation. Biol Blood Marrow Transplant. 2012;18(1 Suppl):S139-S150. doi:10.1016/j.bbmt.2011.10.005

7. Bhatia S, Ramsay NK, Steinbuch M, et al. Malignant neoplasms following bone marrow transplantation. Blood. 1996;87(9):3633-3639. doi:10.1146/annurev.ecolsys.37.091305.110136

8. Kolb HJ, Socié G, Duell T, et al. Malignant neoplasms in long-term survivors of bone marrow transplantation. Ann Intern Med. 1999;131(10): 738-744. doi:10.7326/0003-4819-131-10-199911160-00004

9. Kulkarni S, Powles R, Treleaven J, et al. Melphalan/TBI is not more carcinogenic than cyclophosphamide/TBI for transplant conditioning: Follow-up of 725 patients from a single centre over a period of 26 years. Bone Marrow Transplant. 2000;25(4):365-370. doi:10.1038/ sj.bmt. 1702148

10. Bhatia $S$, Louie $A D$, Bhatia $R$, et al. Solid cancers after bone marrow transplantation. J Clin Oncol. 2001;19(2):464-471. doi:10.1200/JCO. 2001.19.2.464

11. Curtis RE, Metayer C, Rizzo JD, et al. Impact of chronic GVHD therapy on the development of squamous-cell cancers after hematopoietic stem-cell transplantation: An international case-control study. Blood. 2005;105(10):3802-3811. doi:10.1182/blood-2004-09-3411

12. Shimada K, Yokozawa T, Atsuta Y, et al. Solid tumors after hematopoietic stem cell transplantation in Japan: Incidence, risk factors and prognosis. Bone Marrow Transplant. 2005;36(2):115-121. doi:10.1038/ sj.bmt. 1705020

13. Hasegawa W, Pond GR, Rifkind JT, et al. Long-term follow-up of secondary malignancies in adults after allogeneic bone marrow transplantation. Bone Marrow Transplant. 2005;35(1):51-55. doi:10.1038/ sj.bmt.1704706
14. Brown JR, Yeckes $\mathrm{H}$, Friedberg JW, et al. Increasing incidence of late second malignancies after conditioning with cyclophosphamide and total-body irradiation and autologous bone marrow transplantation for non-Hodgkin's lymphoma. J Clin Oncol. 2005;23(10):2208-2214. doi:10.1200/JCO.2005.05.158

15. Leisenring W, Friedman DL, Flowers MED, Schwartz JL, Deeg HJ. Nonmelanoma skin and mucosal cancers after hematopoietic cell transplantation. J Clin Oncol. 2006;24(7):1119-1126. doi:10.1200/JCO.2005. 02.7052

16. Cavalier M, Shmalo JA, Yu M, Billings SD, Abonour R, Nelson RP. Skin cancer after nonmyeloablative hematopoietic cell transplantation. Bone Marrow Transplant. 2006;37(12):1103-1108. doi:10.1038/sj.bmt.1705362

17. Gallagher G, Forrest DL. Second solid cancers after allogeneic hematopoietic stem cell transplantation. Cancer. 2007;109(1):84-92. doi:10. $1002 /$ cncr.22375

18. Schwartz JL, Kopecky KJ, Mathes RW, Leisenring WM, Friedman DL, Deeg HJ. Basal cell skin cancer after total-body irradiation and hematopoietic cell transplantation. Radiat Res. 2009;171(2):155-163. doi:10. 1667/RR1469.1

19. Rizzo JD, Curtis RE, Sobocinski KA, et al. Solid cancers after allogeneic hematopoietic cell transplantation. Blood. 2009;113(5):1175-1183. doi:10.1182/blood-2008-05-158782

20. Abou-Mourad YR, Lau BC, Barnett MJ, et al. Long-term outcome after allo-SCT: Close follow-up on a large cohort treated with myeloablative regimens. Bone Marrow Transplant. 2010;45(2):295-302. doi:10.1038/bmt.2009.128

21. Chen MH, Chang PM, Li WY, et al. High incidence of oral squamous cell carcinoma independent of HPV infection after allogeneic hematopoietic SCT in Taiwan. Bone Marrow Transplant. 2011;46(4):567-572. doi:10.1038/bmt.2010.163

22. Majhail NS, Brazauskas R, Rizzo JD, et al. Secondary solid cancers after allogeneic hematopoietic cell transplantation using busulfan-cyclophosphamide conditioning. Blood. 2011;117(1):316-322. doi:10.1182/ blood-2010-07-294629

23. Yokota A, Ozawa S, Masanori T, et al; Kanto Study Group for Cell Therapy (KSGCT). Secondary solid tumors after allogeneic hematopoietic SCT in Japan. Bone Marrow Transplant. 2012;47(1):95-100. doi:10.1038/ bmt.2011.23

24. Shimoni A, Shem-Tov N, Chetrit A, et al. Secondary malignancies after allogeneic stem-cell transplantation in the era of reduced-intensity conditioning: The incidence is not reduced. Leukemia. 2013; 27(4):829-835. doi:10.1038/leu.2012.299

25. Krishnan AY, Mei M, Sun CL, et al. Second primary malignancies after autologous hematopoietic cell transplantation for multiple myeloma. Biol Blood Marrow Transplant. 2013;19(2):260-265. doi:10.1016/j. bbmt.2012.09.023

26. Bilmon IA, Ashton LJ, Le Marsney RE, et al; CAST Study Group. Second cancer risk in adults receiving autologous haematopoietic SCT for cancer: A population-based cohort study. Bone Marrow Transplant. 2014;49(5):691-698. doi:10.1038/bmt.2014.13

27. Atsuta Y, Suzuki R, Yamashita T, et al; Japan Society for Hematopoietic Cell Transplantation. Continuing increased risk of oral/esophageal cancer after allogeneic hematopoietic stem cell transplantation in adults in association with chronic graft-versus-host disease. Ann Oncol. 2014;25(2):435-441. doi:10.1093/annonc/mdt558

28. Omland SH, Gniadecki R, Hædersdal M, Helweg-Larsen J, Omland LH. Skin cancer risk in hematopoietic stem cell transplant recipients compared with background population and renal transplant recipients. JAMA Dermatol. 2016;152(2):177-183. doi:10.1001/jamadermatol. 2015.3902

29. Michelis FV, Kotchetkov R, Grunwald RM, et al. Long-term incidence of secondary malignancies after allogeneic hematopoietic cell transplantation: A single-center experience. Biol Blood Marrow Transplant. 2017;23(6):945-951. doi:10.1016/j.bbmt.2017.02.015

30. Song JS, London WB, Hawryluk EB, et al. Risk of melanocytic nevi and nonmelanoma skin cancer in children after allogeneic hematopoietic stem cell transplantation. Bone Marrow Transplant. 2017;52(7): 989-997. doi:10.1038/bmt.2017.57

31. Inamoto Y, Matsuda T, Tabuchi K, et al; Japan Society for Hematopoietic Cell Transplantation Late Effects and Quality of Life Working Group. Outcomes of patients who developed subsequent solid cancer after hematopoietic cell transplantation. Blood Adv. 2018;2(15):1901-1913. doi:10.1182/bloodadvances.2018020966 
32. DePry JL, Vyas R, Lazarus HM, Caimi PF, Gerstenblith MR, Bordeaux JS. Cutaneous malignant neoplasms in hematopoietic cell transplant recipients. JAMA Dermatol. 2015;151(7):775-782. doi:10.1001/jama dermatol.2015.121

33. Majhail NS. Secondary cancers following allogeneic haematopoietic cell transplantation in adults. Br J Haematol. 2011;154(3):301-310. doi:10.1111/j.1365-2141.2011.08756.x

34. Deeg HJ, Socié G, Schoch G, et al. Malignancies after marrow transplantation for aplastic anemia and fanconi anemia: A joint Seattle and Paris analysis of results in 700 patients. Blood. 1996;87(1):386-392.

35. Rangwala S, Tsai KY. Roles of the immune system in skin cancer. Br J Dermatol. 2011;165(5):953-965. doi:10.1111/j.1365-2133.2011.10507.x

36. Sloand EM, Pfannes L, Ling C, et al. Graft-versus-host disease: Role of inflammation in the development of chromosomal abnormalities of keratinocytes. Biol Blood Marrow Transplant. 2010;16(12):1665-1673. doi:10.1016/j.bbmt.2010.07.014

37. Vassallo C, Brazzelli V, Alessandrino PE, et al. Normal-looking skin in oncohaematological patients after allogenic bone marrow transplantation is not normal. Br J Dermatol. 2004;151(3):579-586. doi:10.1111/ j.1365-2133.2004.06072.x

38. Rambhia PH, Conic RZ, Atanaskova-Mesinkovska N, Piliang M, Bergfeld WF. Role of graft-versus-host disease in the development of secondary skin cancers in hematopoietic stem cell transplant recipients: A meta-analysis. J Am Acad Dermatol. 2018;79(2):378-380.e3. doi:10.1016/j.jaad.2018.01.012

39. O'Donovan P. Azathioprine and UVA light generate mutagenic oxidative DNA damage. Science. 2005;309(5742):1871-1874. doi:10.1126/ science. 1114233

40. Hojo M, Morimoto T, Maluccio M, et al. Cyclosporine induces cancer progression by a cell-autonomous mechanism. Nature. 1999; 397(6719):530-534. doi:10.1038/17401

41. Wojenski DJ, Bartoo GT, Merten JA, et al. Voriconazole exposure and the risk of cutaneous squamous cell carcinoma in allogeneic hematopoietic stem cell transplant patients. Transpl Infect Dis. 2015;17(2): 250-258. doi:10.1111/tid.12367

42. Epaulard O, Villier C, Ravaud P, et al. A multistep voriconazole-related phototoxic pathway may lead to skin carcinoma: Results from a French nationwide study. Clin Infect Dis. 2013;57(12):182-188. doi:10. 1093/cid/cit600

43. Meyer T, Arndt R, Nind I, Ulrich C, Christophers E, Stockfleth E. Association of human papillomavirus infections with cutaneous tumors in immunosuppressed patients. Transp/ Int. 2003;16(3):146-153. doi:10.1111/j.1432-2277.2003.tb00278.x

44. Andreani V, Richard MA, Blaise D, Gouvernet J, Grob JJ. Naevi in allogeneic bone marrow transplantation recipients: The effect of graftversus-host disease on naevi. Br J Dermatol. 2002;147(3):433-441. doi:10.1046/j.1365-2133.2002.04748.x
45. Feily A, Namazi MR. Why is graft-versus-host disease sometimes associated with leukoderma and fewer melanocytic naevi? J Cosmet Dermatol. 2010;9(1):64-65. doi:10.1111/j.1473-2165.2010.00482.x

46. Socié $G$, Henry-Amar $M$, Devergie $A$, et al. Poor clinical outcome of patients developing malignant solid tumors after bone marrow transplantation for severe aplastic anemia. Leuk Lymphoma. 1992; 7(5-6):419-423. doi:10.3109/10428199209049797

47. Brown SJ, Jackson GH, Reynolds NJ, Lawrence CM. Chronic cutaneous graft-versus-host disease associated with multiple cutaneous squamous cell carcinomas. Clin Exp Dermatol. 2006;31(3):472-473. doi:10.1111/j.1365-2230.2006.02109.x

48. Altman JS, Adler SS. Development of multiple cutaneous squamous cell carcinomas during PUVA treatment for chronic graft-versus-host disease. J Am Acad Dermatol. 1994;31(3 Pt 1):505-507. doi:10.1016/ S0190-9622(09)80014-5

49. Perez HC, Benavides X, Perez JS, et al. Basic aspects of the pathogenesis and prevention of non-melanoma skin cancer in solid organ transplant recipients: A review. Int J Dermatol. 2017;56(4):370-378. doi:10.1111/ijd.13409

50. Howard MD, Su JC, Chong AH. Skin cancer following solid organ transplantation: A review of risk factors and models of care. Am J Clin Dermatol. 2018;19(4):585-597. doi:10.1007/s40257-018-0355-8

51. de Fijter JW. Cancer and mTOR Inhibitors in transplant recipients. Transplantation. 2018;101(1):45-55. doi:10.1097/TP.0000000000001447

52. D'Souza A, Lee S, Zhu X, Pasquini M. Current use and trends in hematopoietic cell transplantation in the United States. Biol Blood Marrow Transplant. 2017;23(9):1417-1421. doi:10.1016/j.bbmt.2017.05.035

53. Majhail NS, Rizzo JD, Lee SJ, et al. Recommended screening and preventive practices for long-term survivors after hematopoietic cell transplantation. Bone Marrow Transplant. 2012;47(3):337-341. doi:10. 1038/bmt.2012.5

54. Inamoto $Y$, Shah NN, Savani BN, et al. Secondary solid cancer screening following hematopoietic cell transplantation. Bone Marrow Transplant. 2015;50(8):1013-1023. doi:10.1038/bmt.2015.63

55. Marks C, Stadler M, Häusermann P, et al. German-Austrian-Swiss Consensus Conference on clinical practice in chronic graft-versus-host disease (GVHD): Guidance for supportive therapy of chronic cutaneous and musculoskeletal GVHD. Br J Dermatol. 2011;165(1):18-29. doi:10.1111/j.1365-2133.2011.10360.x

56. Matin RN, Danby R, Gibbons E, et al. Developing a dedicated dermatology service for allogeneic bone marrow transplant recipients. $\mathrm{Br}$ J Dermatol. 2017;177(6):1758-1759. doi:10.1111/bjd.15343

57. Savani BN, Griffith ML, Jagasia S, Lee SJ. How I treat late effects in adults after allogeneic stem cell transplantation. Blood. 2011;117(11): 3002-3009. doi:10.1182/blood-2010-10-263095

58. Bishop MM, Lee SJ, Beaumont JL, et al. The preventive health behaviors of long-term survivors of cancer and hematopoietic stem cell transplantation compared with matched controls. Biol Blood Marrow Transplant. 2010;16(2):207-214. doi:10.1016/j.bbmt.2009.09.015 